\title{
Anemia Hemolítica por carencia de vitamina "E" en recién nacido de bajo peso de nacimiento. I. Influencia de la alimentación: LM y NAN
}

\author{
Dr. Raúl Corrales,** Dra. Mireya Bravo, ${ }^{*}$ Q.F. Olga Puratic,* T.M. Ana María Dupré.*
}

\begin{abstract}
Henrlytic anemia due to vitamin $\mathbf{E}$ (tocopherol) deficiency in newborns of very low birthweight fed with artificial milk is a well recognized fact, with a different frequency depending on the mlk artificial formula. There is no satisfactory informution related with matemal milk feeding.

We studied prospectively in the newhors unit at the Raberto de] Rio Hospital, newhorns of very low birthweight fed with matemal milk and artificial formula (N.AN).

Thirty seven newboms with less than $1500 \mathrm{~g}$ of birthweight were studied. The $y$ were divided in wo groups: Nine premature infants were fed with manualy obtained matemal milk and twenty eight received. VAX, since the first day of life.

No one of the nise infants fed with maternal milk developed hemolytic anemia due to vitarnin E deficiency. In the group fed with artificial formulu 60.71\%. 17 of 28 infants. developed it, and the diagnosis was made in $64.7 \%$ of them between the forth and sixth week of life.

The explanation for this seems to be a diference in the contents of poliunsaturated tatty acid, Iron. Selenium and Vitarnin $E$ in the milk artilicial formula and matemal milk.
\end{abstract}

Desde 1967 en que Oski y Bamess ${ }^{1}$ describen Jos primeros casos de Anemia Hemolítica por carencia de Vitamina $E$ (tocoferol) en Recièn Nacidos de Pretérmino, hay numerosas publicaciones llamando la atención sobre esta forma de anemia hemolítica.

Se presenta como fenómeno autolimitado entre la $4,{ }^{\text {a }}$ y $6 .^{\text {à }}$ semana de vida en los recién nacidos de bajo peso de nacimiento que reciben alimentación artificial. Se caracteriza por tener niveles de hemoglobina más bajos que los esperados para la edad y peso de nacimiento, alteraciones morfológićas (anisocitosis, poiquilocitosis, glóbulos rojos fragmentados y eventualmente microesferocitos),

\footnotetext{
*Sección Hematología. Departamento y Servicio de Pediatria Hospital Roberto del Río.

**Departamento de Farmacología y Departamento de Pediatría, Facultad de Medicina Norte. Universidad de Chile.
}

respuesta reticulocitaria y aumento del número de plaquetas.

Además de los hallazgos hematológicos se encuentra aumento del porcentaje de hemólisis de glóbulos rojos medido por el test de hemólisis por peróxido de hidrógeno, valor que se ha visto se correlaciona con los bajos niveles plasmáticos de Vitamina E. 1,2,3,4

En el mecanismo de producción hay que considerar diversos aspectos. Por un lado, el rol metabólico de la Vitamina $E$ como protector de membranas biológicas frente a la oxidación de lípidos.

Por otra parte, influye el bajo contenido de Vitamina $\mathbf{E}$ en los niños de bajo peso, que los hace depender del aporte externo poco después del nacimiento. Las leches, incluyendo la materna, tienen un contenido bajo de esta vitamina.

Los requerimientos de Vitamina $\mathrm{E}$ dependen a su vez del contenido de ácidos grasos poliinsaturados (PUFA) de la dieta. Cuando éste es alto se 
produce un cambio en la composición de ácidos grasos de la membrana celular, especialmente del glóbulo rojo, haciéndolo susceptible al daño secundario a la peroxidación de lípidos y aumentando así los requerimientos de Vitamina $\mathbf{E}$.

Esta situación hace que se agrave la condición de carencia del niño de bajo peso, lo que se traduce en mayor peroxidación de los lípidos de la membrana del glóbulo rojo y anemia hemolítica. Esta anemia responde a la administración de Vitamina $\mathrm{E}^{5,6}$

Se han reconocido como factores predisponentes la inmadurez, ${ }^{7}$ el tipo de alimentación recibida por el Recién Nacido y la administración de sustancias oxidantes, ya sea de $\mathrm{O}_{2} \mathrm{u}$ otros elementos como el Hierro. $\$$. 911

Debido a que no se ha correlacionado claramente la diferencia en frecuencia de presentación de esta forma de anemia en nin̄os alimentados con leche materna y artificial, decidimos estudiar su incidencia y edad de aparición con ambas alimentaciones y precisar además los elementos útiles para hacer el diagnóstico, en un grupo de Recién Nacidos de bajo peso de nacimiento del Servicio de Recién Nacidos del Hospital Roberto del Rio.

\section{MATERLAL Y METODO}

Se estudiaron 37 recién nacidos con peso de nacimiento inferior a $1500 \mathrm{~g}$. De éstos, 29 son adecuados para la edad gestacional (AEG) y 8 son pequeños para la edad gestacional (PEG), de estos últimos uno es de término. Las edades gestacionales del resto fluctuaron entre 26 y 35 semanas. Se excluyeron: a) aquellos recién nacidos con incompatibilidad ABO o $\mathrm{RH}$; b) los que a la semana de vida tienen valores de hemoglobina menores de $13 \mathrm{~g} \%$; c) aquellos que necesitaron alguna intervención quirúrgica; y d) quienes presentaron una infección grave o síndrome diarreico agudo.

Los recién nacidos se dividieron en 2 grupos, uno que se alimentó con leche materna exclusivamente, formado por 9 recién nacidos y otro grupo que se alimentó solamente con leche materni- zada (NAN), formado por 28 recién nacidos. A ambos grupos se les hicieron controles de hematocrito $\mathrm{y}$ reticulocitos al ingreso, a la primera, cuarta, sexta, octaba y décima semana de vida. Además se estudió la morfología de glóbulos rojos, (11) recuento de plaquetas (11) y fragilidad de glóbulos rojos frente al peróxido de hidrógeno (12).

Para el diagnóstico de anemia hemolítica se exigió un test de hemólisis por peróxido de hidrógeno de $20 \%$ o más, un descenso de Hemoglobina acompañado de una respuesta reticulocitaria y morfología compatible, especialmente la presencia de poiquilocitosis.

\section{RESULTADOS}

De los recién nacidos alimentados con leche matema ninguno reunió los parámetros exigidos para el diagnóstico, en cambio reunieron los criterios 17 de los $28(60.71 \%$ ) recién nacidos alimentados con NAN.

Otro de los objetivos del trabajo era precisar la edad de presentación de esta forma de anemia hemolítica. Se observó que de los 17 recién nacidos que presentaron anemia hemolítica, en 6 se observó a las 4 semanas $(35,29 \%), 7$ a las 6 semanas $(41.18 \%)$ y 4 a las 8 semanas $(23.53 \%)$. El $76 \%$ presentó la anemia hemolítica entre la $4 .^{\mathrm{a}}$ y la 6 . $^{\text {a }}$ semana de vida.

Los valores promedios \pm 1 D.S. de Hb, porcentaje de hemólisis por $\mathrm{H}_{2} \mathrm{O}_{2}$ se pueden observar en la Tabla I.

En la Fig. N. ${ }^{0}$ l se observa la evolución de la hemoglobina a la $4 .^{a}$ y $6 .^{\text {a }}$ semana en ambos grupos, los valores promedios de hemoglobina son poco más bajos en el grupo alimentado con NAN, diferencia que no fue estadísticamente significativa a la 4. ${ }^{\text {a }}$ y 6 a $^{\text {a }}$ semana. La evolución de los valores promedio de hemoglobina es más baja con NAN que con leche materna.

En la Fig. N. ${ }^{\circ} 2$ se observa la evolución del test de hemólisis por peróxido de hidrógeno a la $4 .^{a}$ y

Tabla ]

\begin{tabular}{lrcrr} 
& \multicolumn{2}{c}{ Ebdad: 4 semanas } & \multicolumn{2}{c}{ Edad: 6 semanas } \\
& Hemólisis \% & Hb g\% & Hemólisis \% \\
\hline L.M. & $11.90 \pm 0.56$ & $3.33 \pm 1.54$ & $9.93 \pm 0.98$ & $1.47 \pm 0.41$ \\
NAN & $10.59 \pm 0.42$ & $22.60 \pm 3.92$ & $8.81 \pm 0.25$ & $24.38 \pm 5.63$ \\
\hline
\end{tabular}


Figura 1

Evolución de Hemoglobina

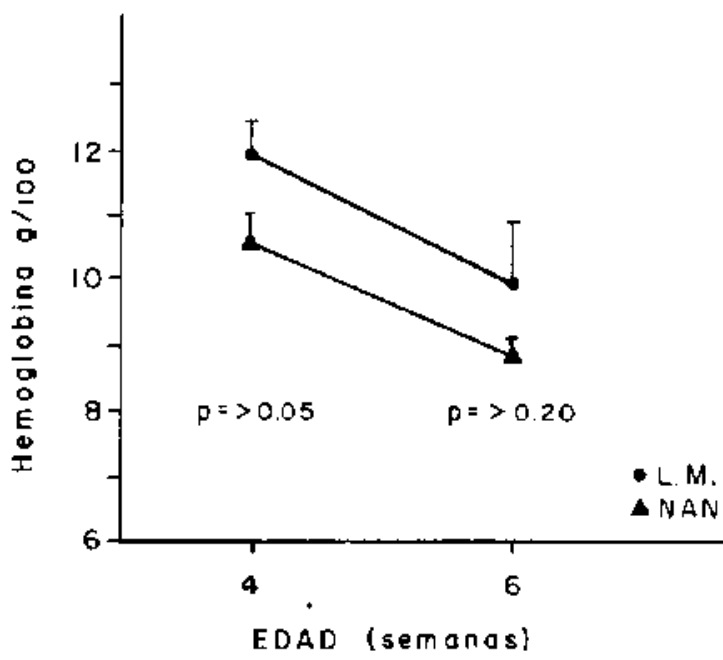

Figura 2

Evolución del test de Hemótisis por $\mathrm{H}_{2} \mathrm{O}_{2}$

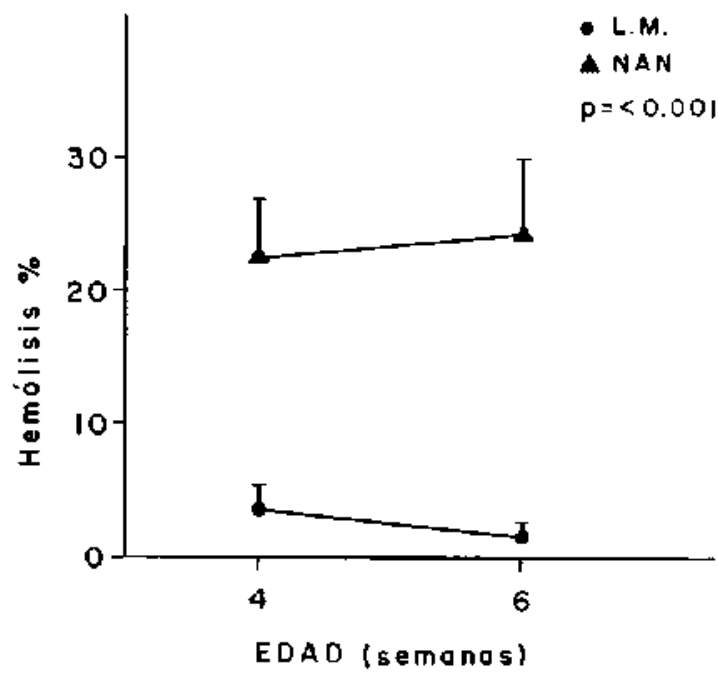

6. semana en ambos grupos. Se puede apreciar que los valores promedio en los recién nacidos alimentados con leche matema son todos bajos, en cambio en los alimentados con NAN el valor promedio es mayor de $20 \%$, la diferencia entre estos promedios es altamente significativa a la 4 . $^{\text {a }}$ y 6 . $^{\text {a }}$ semana $(\mathrm{p}<0.001)$.

En la Fig. N. ${ }^{\circ} 3$ se correlacionan los valores de hemoglobina con el porcentaje de hemólisis por peróxido de hidrógeno en el grupo alimentado con leche materna a la 4 . $^{\text {a }}$ semana, se pueden ob- servar valores de hemólisis similares con distintos valores de hemoglobina, calculado el índice de correlación " $r$ " es -0.67 con un $p<0.10$.

En la Fig. N. 4 se correlacionan los mismos parámetros en el grupo alimentado con NAN a la 4. ${ }^{\text {a }}$ semana. Se pueden apreciar valores de hemólisis por peróxido de hidrógeno similares para los distintos valores de hemoglobina, calculado el índice de correlación " $r$ " es -0.04 con un $p<0.9$.

Junto con estos parámetros se analizó la correlación del test de hemólisis por peróxido de hidrógeno con el porcentaje de reticulocitos y con las alteraciones morfológicas, especialmente poiquilocitosis, al igual que con hemoglobina no se encontró correlación.

Figura 3

Correlación Hb/Hemolisis por $\mathrm{H}_{2} \mathrm{O}_{2}$

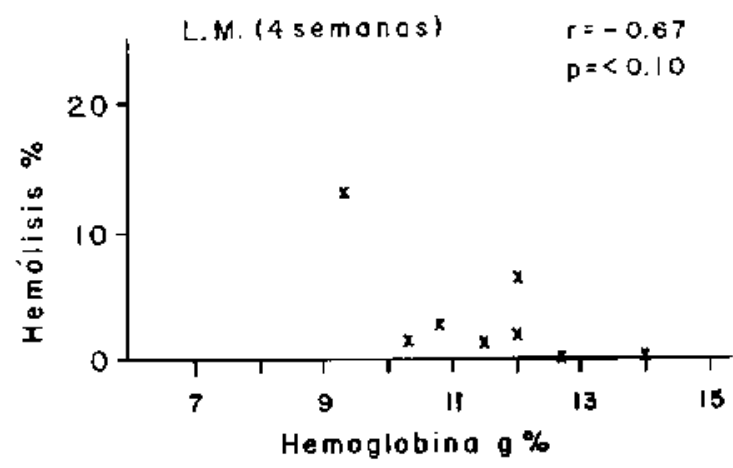

Figura 4

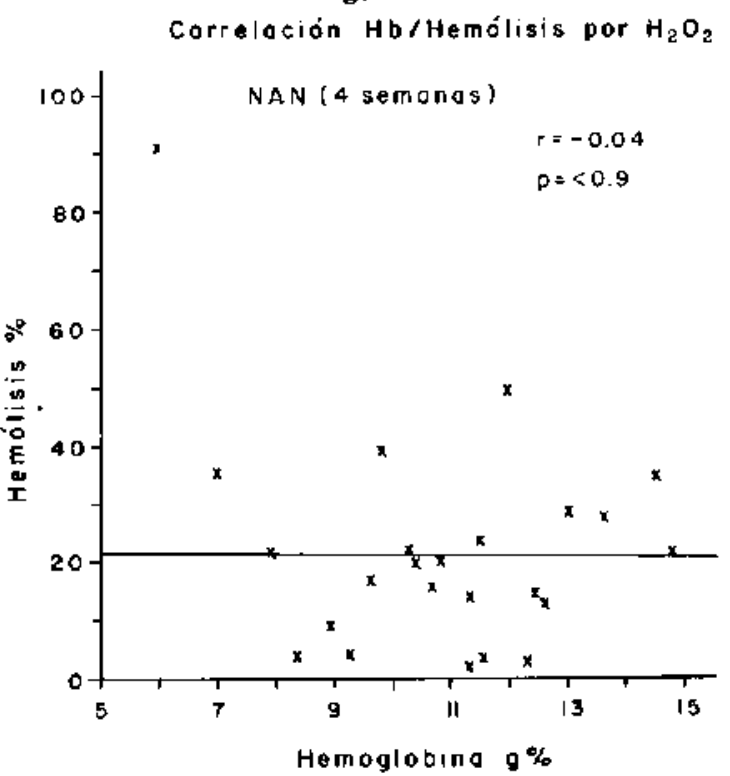




\section{COMENTARIO}

La carencia de Vitamina E con el $\mathrm{RN}$ de bajo peso de nacimiento se manifiesta como anemia hemolítica bajo clertas circunstancias corno son: aumento del contenido de ácidos grasos poliinsaturados de la dieta, administración de sustratos potencialmente oxidantes y presencia de un agente oxidativo extemo.

El tipo de alimentación administrada es por lo tanto uno de los elementos más importantes a considerar al respecto.

Los trabajos de Melhorn y Gross ${ }^{x} y$ Williams $y$ col. ${ }^{9}$ han demostrado que existe una correlación estadísticamente significativa entre porcentaje de ácidos grasos poliinsaturados de la dieta y contenido de Hierro, con el desencadenamiento de una anemia hemolitica.

En el Hospital Roberto del hío los niños de bajo peso de nacimiento son alimentados regularmente con leche matema o NAN.

EI NAV es una fómmula en polvo, que una vez reconstituida en la proporción de agua correspondiente ofrece un alimento de composición cuantitabivamente y cualitativamente semejante a la de la leche matema. De esta forma, teóricamente no deberia haber diferencias significativas en el desencadenamiento de anemia hemolítica.

Los resultados encontrados, sin embargo, muestran diferencias estadisticamente significativas entre los niños alimentados con leche materma y NAN.

Parece lógico analizar en primer lugar las posjbles diferencias en estas leches con los elementos que probadamente han desencadenado anemia hemolítica como son el contenido de ácidos grasos poljinsaturados y el de Hierro.

En la grasa de la leche materna la proporción de ácidos grasos saturados e insahurados es de 46: 48 y en el NAN es 48: 46. La composición de grasas del NAN es lograda con una mezcla formada por $80 \%$ de grasa de leche de vaca y $20 \%$ de aceite de maíz. Con esta mezcla, el ácido linoleico constituye el $12,8 \%$ del total de ácidos grasos, En la leche materna éste es de un $10.6 \%$.

El porcentaje de absorción de grasas y la velocidad de absorción también guardan analogia con la leche materna, aun tomando en cuenta la limitación de absorción de grasas en los niños de bajo peso de nacimiento. E] contenido de hierro del NAN es de $6.0 \mathrm{mg}$ por $100 \mathrm{~g}$ de polvo, lo que en dilución al $13 \%$ da un contenido de $0.78 \times 100 \mathrm{ml}$; la leche matema tiene un contenido promedio de $0.15 \mathrm{mg} \mathrm{x} 100 \mathrm{ml}$.

Cuesta aceptar que estas diferencias en contenido de ácido linoleico y Hierro expliquen diferencias tan grandes como las obtenidas en este estudio.

Otras posibles explicaciones podrian estar en las diferencias en contenidu de tocoferol y selenio entre ambas leches.

El NA. $\mathrm{C}$ contiene $5 \mathrm{mg}$ de Viramina $E$ por $\mathbf{l}(00 \mathrm{~g}$ de polvo, lo que da un contenido de 0.65 mg $x 100$ Inl; en la leche humana éste es de $0.2-0.6 \mathrm{mg} x$ $100 \mathrm{ml}$. Si bien cuantitativamente es semejante, es posible que la absorción del producto sea diferente.

En $1973^{1.3}$ se demostró que el Selenio era una parte integral de la glutathion peroxidasa; esta enzima juega un rol en la protección de la membrana celular contra la degeneración oxidativa como lo hace la vitamina $E, y$ se ha visto que una carencia de Selenio per se puede producir alteraciones semejantes a la carencia de vitamina $E^{14,15}$ 16

El contenido promedio de Se de la leche materna es de $28 \pm 6 \mathrm{ng} / \mathrm{ml}$ y el de las leches de vaca alrededor de la mitad, dependiendo del contenido de Se de los suelos.

La leche humana no sólo tiene un mayor contenido de be y tocoferol que la leche de vaca, sino que además la absorción es mayor y más rápida por lo menos en lo que respecta a tocoferol.

Es posible que todas estas diferencias en conjunto expliquen los resultados obtenidos; pero habría que precisarlo. Mientras esto no se determine, creemos debe usarse Vitamina $\mathbf{E}$ en forma profiláctica en los RN de bajo peso de nacimiento alimentados con NAN.

La edad en cue esta anemia se presentó esta de acuerdo con lo encontrado en la literatura; es decir, es un fenómeno que se presenta a partir de las 4 semanas de vida. En nuestra experiencia la mayor frecuencia se encuentra entre la $4 .^{\text {a }}$ y $6 .^{\text {a }}$ semanas $(76 \%)$. Como se sabe, es un fenómeno autolimitado y tiene que ver probablemente con la mejor absorción de Vitamina $E$ a medida que se produce inaduración. ${ }^{7}$

Basados en la experiencia adquirida creemos que los elementos importantes a considerar para plantear el diagnóstico de anemia hemolítica por carencia de Vitamina $E$ son:

1. Niño de bajo peso de nacimiento con alimentación artificial de 4-8 semanas de vida. 
2. Baja progresiva de valores de $\mathrm{Hb}$ y $\mathrm{Ht}$ acompañada de respuesta reticulocitaria, alteraciones morfológicas de glóbulos rojos (caracterizada por poiquilocitosis, glóbulos rọjos fràgmentados, uno que otro microesterocito) y aumento en el número de plaquetas.

3. Test de hemólisis por peróxido de Hidrógeno sobre $20 \%$.

El análisis estadístico de estos parámetros aislados nos mostró que no hay correlación entre ellos. por esto se hace necesario para plantear el diagnóstico su presencia simultánea. Sin embargo, para nosotros tiene especial valor el test de hemólisis por peróxido de Hidrogeno que se ha demostrado tiene una correlación inversa estadísticamente significativa con los niveles plasmáticos de tocoferol. El test de hemólisis por peróxido de Hidrógeno da una medida de la capacidad antioxidante de un individuo, ya que la capacidad de protección antioxidante disponible está retlejada en el grado en el cual se hemolizan los glóbulos rojus cuando se exponen a peróxido de Hidrógeno. Sin embargo se ha sugerido que anormalidades en este test pudieran ser no especificas sino pueden retlejar también deficiencias asociadas de glutathion peroxidasa o catalas a. ${ }^{\text {? }}$

4. El nivel de tocoferol plasmático sin duda es de la mayor importancia en _.ite tipo de enfermos. Permitiría diferenciar las formas de anemia hemolítica por carencia de Vitamina E de las asociadas a carencia de Se.

\section{RESUMEN Y CONCLUSIONES}

1. En nuestra casuística y con la metodología usada no encontramos anemia hemolítica por carencia de Vitamina $\mathbf{E}$ en los recién nacidos de bajo peso de nacimiento alimentados con leche materna, en cambio la encontranos en un $60.71 \%$ de los recién nacidos alimentados con NAN.

2. Si bien esta forma de anemia hemolítica se presentó entre la $4 .{ }^{i}$ y 8 ." semanas, de vidat, el $76 \%$ de los casos lo hizo entre la $4 .^{\prime \prime}$ y $6 .^{\text {a }}$ semanas, lo que está de acuerdo con lo observado por otros autores.

3. No contando con la determinación de los niveles plasmáticos de tocoferol, para hacer el diagnóstíco son necesarios todos los parámetros utilizados en este trabajo y dentro de éstos es de gran importancia el test de hemólisis.

4. Debido a la alta incidencia de anemiat hemolítica por carencia de vitamina $E$ en los recién uacidos de bajo peso de nacimiento alimentados con NAN, nos parece que la prevención de esta carencia deberia hacerse en forma rutinaria. El ideal seria la administración oral diaria del compuesto hidrosoluble (succituato de d-a tocoferol).

\section{HEFERENCIAS}

${ }^{1}$ Oski, F.A.; Buniess, L.A. Vitamin E deficiency: a previsusily unrecognized cause of hemolytic anemia in the premature infant. J. Pediatr. 70: 211, $196 \overrightarrow{7}$.

${ }^{2}$ Ritchie, J.H., Fish, M.B.; Me Masters, V.; Grossmten, W. Edewa and hemolytis: anemia in premature intants. A Vitam in $\mathrm{E}$ Deticiency Syndrome. N. Engl. J. Med, 279: 1185, 1968.

${ }^{3}$ Lo, S.S.: Frakk, D.; Hitzig, W.H, Vitamis E and hemolytic dnemia in premature infants. Arsh. Dis. Child. 48: 360, 1973.

${ }^{4}$ Dolloman, P R. Iron, vitam in $\mathrm{E}$ and lolate in the pretem inliant. J. Ycdiatr. 85; 742, 1974.

5Janssion, L,.; Holmberg, L.; Velssun, B.i Johansison, B. Yitamin E requirement of preterm infants. Acta Pacdiat. Scand. 67: 45y. 197B.

GGress, S.; Welhorn, D.K. Vitamin E dependent anemia in the premature infant. J. Pediatr. 85: 753, 1974.

T.Me'thern, D.K, Gross, S. Vitanion E dependent anemia in the prenuature intant. 11 Relationships between gestutisnal age and absorption of Vitanin E. J. Pectiatr. 74: 58 L, $197 \mathrm{I}$.

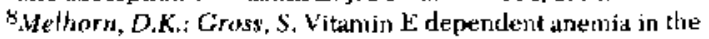
prematire intint. Effectu ol large dases of medicinal iron. I. "ediatr. 79: 569, 1971.

"Williems, M.I.; Shor, R.J.; O'Yejt. P.I.; Oski, F_A. Hole of dietary iron and fat on Vitamin E deticiency anem ia of infancy. N. Engl. J. Med. 292: 887, 1975.

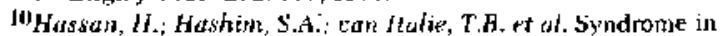
premature infants associated wath low plas nua vitamin $E$ levels athd high polynsisaturated fatty acid diet. Am. J. Clin. Nutr. 19 : 147. 1946 .

"Dacie, J.V,; Lewis, S.M. Practical Hemitology, 4, Ed. 1968. Grune and Stratton Inc. N.Y.

12Hose, C.S.; Gyōrky, P. Speciticin of hemolytic reaction in vitamin E defjeient erytrocytes. An. J. Physiol. 168: 414, 1952.

${ }^{13}$ Rotruck, $J . T$, et al. Selenium: Biochenical role as a coutuponent of gluthathione peroxidase. Science 179 : $5 \$ \$ \$, 1973$.

14 Gruss, S. Antioxidant relationship between velenium dependent gluthathione peroxidase and tocmpherol. Am. J. Pediat. Hematol. Oncol. I: 61, 1979 .

${ }^{15}$ Huga, P.: Lunde, G. Selenium and vitamin $E$ in cord blood from preterm and fill teru infants. Acta Fatycliat. Scand. 67: $735,197 \mathrm{~s}$.

${ }^{16}$ Gross, S. Hernulytic anemia in premature infants; relatjonship to vitamin $\mathbf{E}$, selenium gluthathione peroxidase and erythros cyte lipids. Sern. Hematol. 13: 187, 1976.

17Gross, R.T.; Brneci, R.; Rudolph, N,; Schrieder, E.; Kochen, J.A. Fydrogen peroxide toxictly and detuxifecation in the erytrucytes of newbon infants. Blood 29: 481, 1967 . 